\title{
PROFILE OF PAEDIATRIC MALIGNANCIES: A FIVE YEAR STUDY
}

JABEEN $\mathrm{S}^{1}$, HAQUE $\mathrm{M}^{2}$, ISLAM MJ ${ }^{3}$, TALUKDER $\mathrm{MH}^{4}$

\begin{abstract}
:
Objective: The objective of this study was to find out the profile of childhood cancers in National Institute of Cancer Research and Hospital, (NICRH), Dhaka during 2005 to 2009.

Methodology: It was a retrospective study using hospital based cancer registry records from January 2005 to December 2009. All the children below 15 years with confirmed diagnosis of cancer by means of histological or cytological examinations were included in this study.

Results: There were 28409 new confirmed cases attended out patient department of NICRH during these 5 years. Among which 1250 were below 15 years of age. An average 250 cases attended per year. Overall pediatric tumours were $4.4 \%$ of total cancers. The frequency of cancer was found to be higher among boys (62\%) than girls (38\%) with a ratio of 1.6:1. Majority of the children were from rural areas (67\%) compared to (33\%) from urban areas. The results showed that Lymphoma (24.2\%), Retinoblastoma (17.4\%) and Leukaemia (14.3\%) were the commonly found childhood cancers among the children attended at NICRH during data collection period. Other less commonly found tumor were bone tumour (7.2\%), kidney tumor (6.8\%) Central Nervous System Tumour (3.7\%),Testicular Tumour (3.7\%), and Hepatocellular cancer (1.3\%).

Conclusion: Lymphoma, acute lymphoblastic leukemia and bone tumor commonly found in children above 5 years in contradiction to retinoblastoma, leukaemia and lymphoma which were prevalent in children less than 5 years of age.
\end{abstract}

Key Words: Cancer registry, paediatric malignancies, cancer profile.

J Dhaka Med Coll. 2010; 19(1) : 33-38.

\section{Introduction:}

Worldwide, the annual number of new cases of childhood cancer exceeds 200,000 and more than $80 \%$ of these are from the developing world. ${ }^{1}$ Cancer remains one of the major causes of death in children between the ages of 0 to 14 years. $^{2}$ Paediatric cancers differ markedly from adult cancers in their nature, distribution and prognosis. Paediatric oncologists face unique challenges because treatment with irradiation, surgery and chemotherapy can adversely affect the children's growth and development. The incidence of childhood cancer and type vary greatly throughout the world. Though lower compared with the incidence of some adult cancers, it comes next to accidents as the leading cause of death among children in the developed world. ${ }^{2}$
Cancer is rare in children under 15 , compared to adults; but cancer patterns at a young age present peculiar characteristics and deserve separate analysis.

Unfortunately, good-quality population-level statistics on the occurrence of cancer at young ages have been more difficult to obtain than in adults $^{3}$ and serious under-reporting, even in western countries, has been documented ${ }^{4,5}$.

The patterns of childhood cancer in America and Europe are almost the same, with leukemia and tumors of the central nervous system accounting for over one-half of the new cases. Children in developing nations are increasingly affected by malignancy in addition to rampant malnutrition and infections, but committed registry maintenance of paediatric

1. Assistant Professor, Department of Community Medicine, National Institute of Preventive \& Social Medicine (NIPSOM), Dhaka.

2. Associate Professor, Department of Community Medicine, National Institute of Preventive $\&$ Social Medicine (NIPSOM), Dhaka.

3. Assistant Professor, National Institute of Cancer Research \& Hospital (NICRH), Dhaka.

4. Associate Professor, National Institute of Cancer Research \& Hospital (NICRH), Dhaka.

Correspondence: Dr. Suraiya Jabeen 
tumours is lacking. As many of common childhood malignancies are curable there is a need to have a dedicated pediatric cancer registry for assessing the magnitude of problem in our country. However, there is a dearth of data due to lack of nation wide population based cancer registries on the incidence and patterns of childhood cancer in developing countries. Although many papers have been published on this in some developing countries, ${ }^{6}$ reports on the pattern and incidence of childhood cancer in Bangladesh are very few. The objective of this study was to find out the profile of childhood cancer in National Institute of Cancer Research and Hospital (NICRH), Dhaka, Bangladesh.

\section{Materials and Methods:}

We used last 5 years hospital records in this retrospective study from January 2005 to December 2009. All children with cancer, aged 0 to 14 years diagnosed by means of histological or cytological examination during that period, were included in the study. In NICRH, hospital based cancer registry is running from 2005, under supervision of Department of Cancer Epidemiology. Patients from all over the country attend this government hospital for better care and cure.

The profile of childhood cancer was studied focusing on the prevalence of tumors according to age, sex, rural and urban distribution. Data were processed by editing and post-coding and analyzed by SPSS version 15.0.

\section{Results:}

There were twenty eight thousand four hundred and nine (28409) confirmed new cases attended out patient department of NICRH during January 2005 to December 2009. Among which twelve hundred and fifty (1250) belong to pediatric group (below 15 years of age). An average of 250 cases attended per year. The pediatric tumours comprised of $4.4 \%$ of all malignancies during theses 5 years time. Out of 1250 patients, $62 \%$ were male and $38 \%$ were female with a male: female ratio of 1.6:1.0. Almost all were (92\%) Muslim. Their average family size was $4.6(\mathrm{SD} \pm 2.5334)$ and parent's average monthly family income was TK.8, 129.88 (SD $\pm 2,809.21$ ). Most of the children with cancer came from the rural areas (67\%) compared to $33 \%$ from urban areas (Table-I).
Table- I

Distribution of childhood cancer patients according to place of residence and gender

\begin{tabular}{lccr}
\hline Sex & \multicolumn{2}{c}{ Place of residence } & Total(n\%) \\
\cline { 2 - 3 } & Rural (n\%) & Urban (n\%) & \\
\hline Male & $496(59.5)$ & $279(66.9)$ & $775(62.0)$ \\
Female & $337(40.5)$ & $138(33.1)$ & $475(38.0)$ \\
\hline Total & $833(100.0)$ & $417(100.0)$ & $1250(100.0)$ \\
\hline
\end{tabular}

Year- wise frequency of pediatric tumours was worked out as shown in Fig. 1. There were 191 cancer children in 2005, 222 in 2006, 187 in 2007,259 in 2008 and 391 in 2009 attended NICRH for seeking cancer treatment. There was a gradual rise of paediatric cancer patients during the study period.

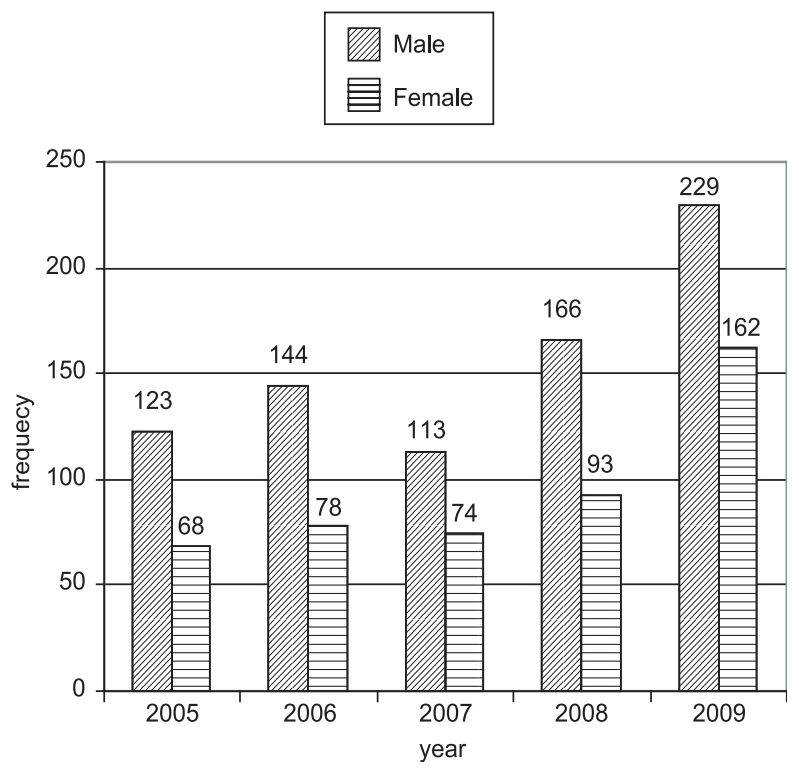

Fig.-1: Multiple bar diagram showing sex of the respondents by year

Tumours were arranged according to patient's age group in 0-4 year, 5-9 year and 10-14 years. The distribution of children according to their age showed that the majority of them belonged to $10-14$ years $(37.7 \%)$, followed by $5-9$ years $(31.4 \%)$ and rest $30.9 \%$ were from $0-4$ years age group. The mean age of the patients was 9.48 ( $\mathrm{SD} \pm 2.66)$ years (Table-II).

Lymphomas were the most prevalent malignancies, accounting for 302 cases $(24.2 \%)$. Among the lymphomas, non-Hodgkin lymphomas (NHL) were more prevalent than Burkitt's lymphoma and Hodgkins lymphoma. 
The prevalence of NHL in males was found to be $65 \%$ while it was $35 \%$ in females. Male preponderance of Hodgkins' lymphoma was $83 \%$ while it was $17 \%$ in females. (Fig.-2)

Retinoblastoma was the second (17.4\%) most commonly found cancer in children attended NICRH during the study period. About 70\% belonged to 5 years or below age group. There was no difference between male to female ratio

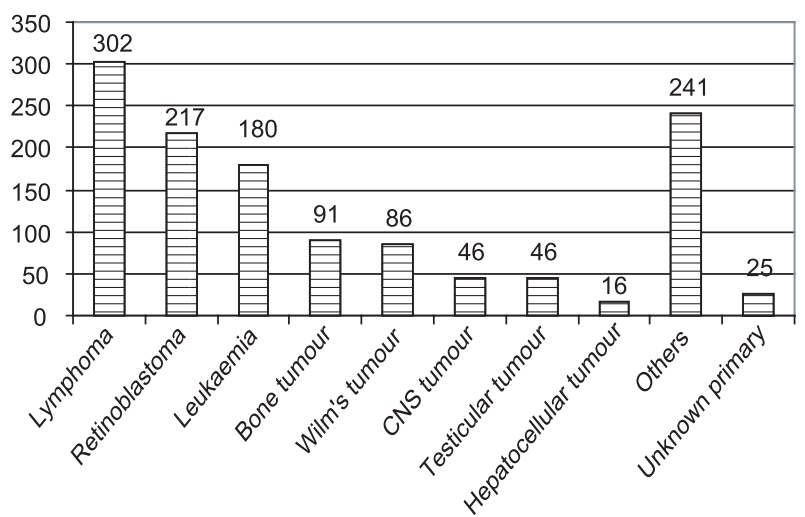

Fig.-2: Paediatric cancer pattern of NICRH from January 2005 to December 2009 for retinoblastoma among our patients, both were almost equal in number. Leukaemia constituted about $14.3 \%$ of all childhood malignancies; among them ALL were commonly found, and almost $77 \%$ sufferers were male. Bone tumour $(7.3 \%)$ was found more common among 10-14 years children. About 52\% sufferers were male patients. Osteogenic sarcomas were more common in male children than their counterparts. Kidney tumour were found $6.8 \%$ among the children and $70 \%$ were male. Majority suffered from Wilm's tumour and was found most commonly in 5-9 years age group. Central nervous system tumours $(3.7 \%)$ were found in all three groups, and here also $73 \%$ sufferers were male .All the cases of Hepatocellular cancer $(1.3 \%)$ were found below the 5 years age group and $83 \%$ were male. (Fig.-2)

The results showed that lymphoma, leukemia and bone tumor commonly occurred in children above five years of age in contradistinction to retinoblastoma, leukaemia and lymphoma commonly occurred in children younger than 5 years. (Table-III)

Table- II

Distribution of childhood cancer according to age group

\begin{tabular}{lcccc}
\hline Types & \multicolumn{3}{c}{ Age (years) } & Total \\
\cline { 2 - 4 } & $0-4$ & $5-9$ & $10-14$ & $302(24.2 \%)$ \\
All Lymphomas & 40 & 98 & 164 & \\
-NHL & 20 & 44 & 109 & \\
-Burkitt's Lymphoma & 7 & 11 & 27 & $217(17.4 \%)$ \\
-Hodgkin's & 10 & 29 & 35 & $180(14.3 \%)$ \\
Lymphoma & & & & \\
Retinoblastoma & 151 & 59 & 7 & \\
All Leukaemias & 82 & 33 & 65 & \\
-Acute Lymphocytic & 69 & 22 & 51 & $91(7.3 \%)$ \\
- Acute Non & 5 & 5 & 9 & $86(6.8 \%)$ \\
Lymphocytic & & & & \\
All Bone & 0 & 39 & 52 & $46(3.7 \%)$ \\
-Osteogenic Sarcoma & & 34 & 79 & \\
All Kidney & 33 & 46 & 6 & \\
-Wilm's Tumour & 31 & 43 & 13 & $46(3.7 \%)$ \\
All Brain \&Spinal & 13 & 20 & 8 & $16(1.3 \%)$ \\
- Astrocytoma & 4 & 11 & 3 & $241(19.3 \%)$ \\
- Medulloblastoma & 6 & 4 & 39 & $25(2.0 \%)$ \\
Testicular Tumour & 0 & 7 & 0 & $1250(100.0)$ \\
Hepatocellular & 16 & 0 & 111 & \\
Others & 40 & 90 & 13 & \\
Unknown primary & 12 & 0 & $471(37.7 \%)$ & \\
Total & $387(30.9 \%)$ & $392(31.4 \%)$ & & \\
\hline
\end{tabular}


Table-III

Distribution of childhood cancer pattern by below and above 5 years age

\begin{tabular}{lcc}
\hline Cancer Pattern & \multicolumn{2}{c}{ Age in years (n \%) } \\
& $<5$ yrs & $>5 y r s$ \\
\hline Retinoblastoma & $151(39.0)$ & $66(7.7)$ \\
Lymphoma & $40(10.3)$ & $262(30.2)$ \\
Leukaemia & $82(21.2)$ & $98(11.5)$ \\
Bone tumour & 0 & $91(10.5)$ \\
Wilm's Tumour & $33(8.5)$ & $53(6.2)$ \\
CNS Tumour & $13(3.4)$ & $33(3.9)$ \\
Testicular Tumour & 0 & $46(5.5)$ \\
Hepatocellular & $16(4.1)$ & 0 \\
Others & $40(10.3)$ & $201(23.3)$ \\
Unknown primary & $12(3.2)$ & $13(1.5)$ \\
\hline Total & $387(100.0)$ & $863(100.0)$ \\
\hline
\end{tabular}

\section{Discussion:}

Incidence of paediatric tumours is on rise ${ }^{3}$. In our study paediatric tumours constituted $4.4 \%$ of all malignancies, our result is almost similar to Rathi et al. ${ }^{7}$, where they found $3.58 \%$ childhood cancers among all malignancies.

But the proportion of childhood cancers seems to be high compared to that of developed countries. In USA, where childhood cancers are $0.8 \%$ of the total, ${ }^{8}$ two possible reasons for this difference could be that our data is from hospital and so is not a true representation of the cancer burden. Moreover, children form a larger part of the population in a developing country where the life expectancy is lower than in the developed world. Majority (92\%) of our respondents was Muslims, out of 1250 patients, $62 \%$ were male and $38 \%$ were female. Male female ratio was 1.6:1.0. This ratio does not accord to our national statistics, but it might be the reflection of our culture where male child is considered as more valuable than female. Their average family size was 4.6 $(\mathrm{SD} \pm 2.5334)$ and parent's average monthly family income was TK.8, 129.88 (SD $\pm 2,809.21$ ). Most of the children with cancer came from the rural areas (67\%) compared to 33\% from urban areas. This result reflects our national statistics. $^{9}$
We found that the three most common cancer groups in our series were lymphomas $(24.2 \%)$, retinoblastomas $(17.4 \%)$ and leukaemias $(14.3 \%)$. This is similar to that reported from North African countries, Morocco. ${ }^{10}$ However, it is in contrast to the data from the developed world, for example, in the $\mathrm{USA}^{8}$ the three most frequent major childhood cancers diagnosed were leukemia $(30.2 \%)$, central nervous system cancers $(21.7 \%)$ and lymphomas (10.9\%).

Lymphomas (302, 24.2\%) were the commonest cancer among the children attended NICRH during the study period. NHL was more frequent than Burkitt's and Hodgkin's lymphomas. NHL constituted $57 \%$ of all lymphomas, Burkitt's lymphoma was about 15\%, and Hodgkin's disease was $24.5 \%$. In some studies, Burkitt's lymphoma has been reported to be around 18$20 \%$ of total lymphomas ${ }^{11}$. In our study of Hodgkin's disease frequency was found higher around 10-14 years age group.

Retinoblastoma was the second most common childhood tumour affecting $17.4 \%$ of study population. Almost $70 \%$ of retinoblastoma at our hospital presented below 5 years of age, and rest 30\% presented late- at ages older than 5 years. Retinoblastoma accounts for $2.5-4 \%$ of all childhood cancers in most developed countries. ${ }^{5}$ This study result accords with the findings observed by Ayodeji ${ }^{12}$, where they found retinoblastoma as second most common malignancies among children. In our study majority of the retinoblastoma cases were referred from different eye hospitals for radiotherapy treatment.

Leukaemia (14.3\%) is the third most common childhood cancer found in NICRH. Almost 79\% of leukamias were acute lymphoblastic leukaemia (ALL). In the Childhood Cancer Report UICC $2006^{13}$ it is found that hematological malignancies were second most common malignancies affecting $18.1 \%$ children. In our study leukaemias were more common in less than 5 years old. Study result similar with the observations made by McKinneya ${ }^{14}$, they found leukaemia commonly among 0-4 years old. Literature review shows acute lymphoblastic leukemia is the most 
common childhood malignancy ${ }^{4}$ but the frequency of leukaemia was lower in our centre, may be our hemato-oncology department started fully only a year back.

Bone tumour comprises $7.3 \%$ of all malignancies among our children. Almost 90\% belonged to osteogenic sarcoma of long bones. This study accords with Mirabello ${ }^{15}$ study, where they reported that osteosarcoma occurs predominantly in adolescents and young adults and osteosarcoma accounts for approximately $5 \%$ of childhood tumors.

Nephroblastoma $(86,6.8 \%)$ was the fifth most common cancer identified in the study; this is comparable with data from other countries. ${ }^{16}$

Brain tumours were not much commonly found malignancy at our institute, affecting only 3.7\% of children in our study. Most common brain tumour was astrocytomas. Majority of astrocytomas occurred in age $>5$ years. In developed countries CNS cancers are the second most common childhood cancer (22$25 \%$ ) and lymphomas a distant third. ${ }^{17}$ In contrast, in India lymphomas often exceed CNS tumours, particularly in males. Interestingly, the incidence of CNS tumours in children in developed world has increased in the last 3040 years with increasing availability of $\mathrm{CT}$ and MRI scanners. 18

The pattern of childhood tumours showed wide variation among the age groups. Frequency of Retinoblastoma, leukaemia and lymphoma was higher in children less than five years and lymphomas, leukaemia and bone tumours were found more in above five years aged children. This result was similar with Huda ${ }^{19}$ where they found lymphoma, acute lymphoblastic leukemia and bone tumor commonly occurred in children above 5 years in contradistinction to kidney tumor and retinoblastoma which was prevalent in children less than 5 years of age. However, our study findings may not reflect our national statistics, as we have collected data from hospital, not from population.

From the study findings, it can be concluded that lymphoma, acute lymphoblastic leukaemia and bone tumour were commonly found in children above five years in contradiction to retinoblastoma, leukaemia and lymphoma which were prevalent in children less than 5 years of age.

\section{References:}

1. Arora RS, Eden T, Kapoor G. Epidemiology of childhood cancer in India. Indian J Cancer. 2009; 46: 264-73.

2. American Cancer Society. Cancer Facts and Figures 2005 reviewed on March 29, 2005 accessed at http://www. cancer.org.

3. Parkin DM, Stiller CA, Draper GJ, Bieber CA. The international incidence of childhood cancer. Int J Cancer. 1988; 42: 511-20.

4 Draper GJ, Bower BD, Darby SC, Doll R. Completeness of registration of childhood leukaemia near nuclear installations and elsewhere in the Oxford region. BMJ. 1993; 299: 952.

5. Cook-Mozaffari P, Ashwood FL, Vincent T, Forman D, Alderson M. Cancer Incidence and Mortality in the Vicinity of Nuclear Installations, England and Wales. 1987; 4(1): 959-80.

6. Akhtar SS, Abu Bakr MA, Dawi SA, Hug IU.. Cancer in Libya - a retrospective study (19811985). Afr J Med Sci. 1993; 22(1): 17-24.

7. Rathi AK, Kumar S, Ashu A, Singh K and Bahadur AK. Epidemiology Of Pediatric Tumours At A Tertiary Care Centre. Indian J Med Paediatr Oncol. 2007; 28(2): 343-50.

8. Ferlay J, Bray F, Pisani P, Parkin DM. GLOBOCAN 2002: Cancer Incidence, Mortality and Prevalence Worldwide. Lyon: IARC Press; 2004.

9. Statistical Pocket Book of Bangladesh 2007. Bangladesh Bureau of Statistics. Planning division, Ministry of Planning, Government of the People's Republic of Bangladesh.

10. Hussain MA, Abass FE, Ahmed HM. Malignant disease in Sudanese children. East Afr Med J. 1988; 65(1): 507-13.

11. Linet MS, Ries LA, Smith MA, Tarone RE, Devesa SS. Cancer surveillance series: recent trends in childhood cancer incidence and mortality in the United States. J Natl Cancer Inst. 1999; 91(4): 1051-8.

12. Ayodeji O, Agboola J, Adekanmbi F A, Musa A A, Sotimehin A S, Deji A M . Pattern of childhood malignant tumours in a teaching hospital in south-western Nigeria. MJA. 2009; 190(1): 12-4. 
13. Childhood Cancer: rising to the challenge. Childhood Cancer Report. UICC. 2006.

14. McKinneya PA, Feltbowera RG, Parslowa RC, Lewisb IJ. Patterns of childhood cancer by ethnic group in Bradford, UK 1974-1997. 2003; 39(1): 92-7.

15. Mirabello L, Troisi RJ, Savage SA: Osteosarcoma incidence and survival rates from 1973 to 2004: data from the Surveillance, Epidemiology, and End Results Program. Cancer. 2009; 115(7): 1531-43.

16. Mandong BM, Angyo IA, Zoakah AI. Paediatric solid malignant tumours in JUTH, Jos (hospital- based histopathology study). Nig J Med. 2000; 9: 52-5.

17. Stiller Ceditor, Childhood cancer in Britain: Incidence survival, mortality. Oxford: Oxford University Press; 2007.

18. Black WC. Increasing incidence of childhood primary malignant brain tumours-enigma or nobrainer? J Natl Cancer Inst. 1998; 90: 1249-51.

19. Huda M. Haroun, Mohamed S, Mahfouz A M, Elhaj. Patterns Of Childhood Cancer In Children Admitted To The Institute Of Nuclear Medicine, Molecular Biology And Oncology. J Fam Comm Med. 2006: 13(2): 71-4. 